\title{
Application of 2D URANS in fluid structure interaction problems of rectangular cylinders
}

\author{
F. Nieto ${ }^{1}$, D. Hargreaves ${ }^{2}$, J. Owen ${ }^{2} \&$ S. Hernández ${ }^{1}$ \\ ${ }^{I}$ School of Civil Engineering, University of La Coruña, Spain \\ ${ }^{2}$ Faculty of Engineering, University of Nottingham, UK
}

\begin{abstract}
In this work the authors have studied the applicability of the 2D URANS approach adopting a block structured mesh and Menter's SST k-omega turbulence model for the aspect ratio 4 rectangular cylinder. As a first step, a verification study is carried out for the mesh refinement level and the time step size. Then, the static response for a $0^{\circ}$ angle of attack is considered for validation purposes. The vortex induced vibration response is computed based on the outof-phase component and phase angle of a one degree of freedom heave forced oscillation simulation at different reduced velocities. Finally, the flutter derivatives are evaluated based on the results of forced induced oscillations in both heave and pitch degrees of freedom. The computational results obtained herein have been in agreement with both experimental and numerical data available in the literature. The proposed methodology based on 2D URANS is less computer power demanding than a 3D LES approach, while it keeps a good accuracy level, which is of particular interest in the frame of industrial applications.
\end{abstract}

Keywords: CFD, rectangular cylinder, URANS, vortex shedding, flutter derivatives.

\section{Introduction}

Fluid structure interaction problems in rectangular cylinders have been extensively studied as test cases in CFD fluid structure interaction problems due to its intrinsic interest, as rectangular members are present in many structures as also the geometry of the cross-section of buildings and bridge decks can be close to the rectangular one, with different aspect ratios. Besides this, it is a simple 
geometry and the profusion of experimental based research allows an accurate validation of the numerical results.

Rectangular prisms show aeroelastic phenomena such as vortex-induced vibration (VIV), torsional flutter, galloping and coupled flutter as it has been described by Takeuchi and Matsumoto [1], and their response is different depending on the width to depth ratio $(B / H)$.

The number of references in the literature regarding experimental studies on rectangular cylinders is plentiful. In the same manner, form 1990 the number of Computer Fluid Dynamics based studies on the response of fixed rectangular cylinders is large and just as a minimal sample the works by Shimada and Ishihara [2] or Bruno et al. [3] are highlighted.

On the other hand, the number of published references dealing with the numerical simulation of Fluid Structure Interaction (FSI) problems is not so extensive. In an early application authored by Murakami et al. [4], the results of forced oscillations and wind-induced free oscillation of a square cylinder using a 3D LES approach were reported. The work led by Tamura [5], Tamura and Itoh [6], and Tamura and Ono [7] on the oscillations of rectangular cylinders and turbulence effects must also be highlighted. Also in Mendes and Branco [8], the computed values for the $H_{1}^{*}$ and $A_{2}^{*}$ flutter derivatives of the $\mathrm{B} / \mathrm{H}=4$ rectangular cylinder are reported employing a $2 \mathrm{D}$ approach with no turbulence model. In [9], the numerical results for the $A_{2}^{*}$ flutter derivative of the $\mathrm{B} / \mathrm{H}=4$ rectangular cylinder using a 3D LES approach are reported. Later, Sun et al. [10] published the complete set of 18 flutter derivatives for the $\mathrm{B} / \mathrm{H}=4$ rectangular cylinder using a 2D URANS (Unsteady Reynolds Averaged Navier-Stokes) modeling and a k- $\omega$ turbulence model; also the effect of the incoming flow level of turbulence was studied. Finally, Shimada and Ishihara [11] reported the responses of ratios 2:1 and 4:1 rectangular prisms subject to forced and free wind-induced oscillations. The authors in the last reference adopted a 2D URANS approach and a modified $k-\varepsilon$ turbulence model to study the aerodynamic instabilities of interest avoiding the burdensome 3D numerical simulations.

The motivation of the current piece of research is to explore the applicability of a 2D URANS approach using Menter's SST k- $\omega$ turbulence model in combination with a low Reynolds strategy in the proximity of the bluff body; which can be accomplished by means of the open source OpenFOAM solver. A block structured mesh has been employed since they are particularly suited for the automatic grid generation in the context of parametric and optimum design problems. The phenomena of interest are the prediction of the VIV as well as the computation of the flutter derivatives of rectangular cylinders. As a case study, the $\mathrm{B} / \mathrm{H}=4$ rectangular cylinder has been chosen due to the existing experimental and computational references in the literature for validation of the results.

\section{Outline of the numerical formulation}

The time averaging of the equations for conservation of mass and momentum gives the Reynolds averaged equations of motion in conservation form [12]: 


$$
\begin{gathered}
\frac{\partial U_{i}}{\partial x_{i}}=0 . \\
\rho \frac{\partial U_{i}}{\partial t}+\rho U_{j} \frac{\partial U_{i}}{\partial x_{j}}=-\frac{\partial P}{\partial x_{i}}+\frac{\partial}{\partial x_{j}}\left(2 \mu S_{i j}-\rho \overline{u_{\imath}^{\prime} u_{\jmath}^{\prime}}\right) .
\end{gathered}
$$

where $U_{i}$ is the mean velocity vector, $x_{i}$ is the position vector, $t$ is the time, $\rho$ is the fluid density, $u_{i}^{\prime}$ is the fluctuating velocity and the over-bar represents the time average, $P$ is the mean pressure, $\mu$ is the fluid viscosity, $S_{i j}$ is the mean strain-rate tensor. From the former equation, the specific Reynolds stress tensor is defined as:

$$
\tau_{i j}=-\overline{u_{\imath}^{\prime} u_{j}^{\prime}},
$$

which is an additional unknown to be modeled based on the Boussinesq assumption for one and two equation turbulence models.

$$
\tau_{i j}=2 v_{T} S_{i j}-\frac{2}{3} k \delta_{i j}
$$

where $v_{T}$ is the kinematic eddy viscosity, $S_{i j}$ is the mean strain-rate tensor and $k$ is the kinetic energy per unit mass of the turbulent fluctuation.

In this work the closure problem is solved applying Menter's k- $\omega$ SST model for incompressible flows [13].

For the simulations where forced oscillations of the bridge deck have been imposed, the Arbitrary Lagrangian Eulerian (ALE) formulation has been applied. The conservation of mass and momentum equations are written as follows [14]:

$$
\begin{gathered}
\frac{\partial\left(U_{i}-U_{g i}\right)}{\partial x_{i}}=0 \\
\rho \frac{\partial U_{i}}{\partial t}+\rho U_{j} \frac{\partial\left(U_{i}-U_{g i}\right)}{\partial x_{j}}=-\frac{\partial P}{\partial x_{i}}+\frac{\partial}{\partial x_{j}}\left(2 \mu S_{i j}-\rho \overline{u_{\imath}^{\prime} u_{\jmath}^{\prime}}\right)
\end{gathered}
$$

where $U_{g i}$ is the grid velocity in the i-th direction.

The forced displacement is imposed at the bluff-body boundary and the mesh control is achieved computing the motion of the grid points solving the Laplace equation with variable diffusivity using a quadratic distance based method.

\section{Forced oscillations in heave}

Forced oscillation of a bluff body allows analyzing its vortex-induced response as well as torsional flutter. Next, a brief summary of the fundamental formulation is going to be presented following [11], whose notation is employed in the following.

Forced displacement is imposed on a bluff body, in a single degree of freedom, according to the following expression for heave: 


$$
h(t)=h_{0} \sin \left(\omega_{m} t\right)
$$

where $h(t)$, positive upwards, represents the forced oscillation in heave, $h_{0}$ is the displacement amplitude, $\omega_{m}$ is the forced vibration angular frequency and $t$ is the time.

The main components of the unsteady wind force acting on the oscillating bluff-body are the vortex-shedding frequency component and the forced frequency component.

The forced oscillation frequency content of the unsteady lift force (or moment) can be written as:

$$
F_{m}(t)=F_{0} \sin \left(\omega_{m} t+\beta\right)
$$

where $F_{0}$ is the amplitude of the unsteady lift at the excitation frequency, and $\beta$ is the phase shift with respect to the forced oscillation.

Applying the Fourier decomposition of the unsteady lift force per unit of span length, $F_{0}$ and $\beta$ can be obtained:

$$
\begin{gathered}
{\left[a_{m}, b_{m}\right]=\frac{1}{T} \int_{-T}^{T} F(t)\left[\cos \omega_{m} t, \sin \omega_{m} t\right] d t} \\
F_{0}=\sqrt{a_{m}^{2}+b_{m}^{2}} \\
\beta=\tan ^{-1}\left(-b_{m} / a_{m}\right)
\end{gathered}
$$

And the forced frequency component of the unsteady lift force acting on the bluff-body is:

$$
F_{m}(t)=F_{0} \cos \beta \sin \left(\omega_{m} t\right)+F_{0} \sin \beta \cos \left(\omega_{m} t\right)
$$

where $F_{m R}=F_{0} \cos \beta$ is the in-phase component and $F_{m I}=F_{0} \sin \beta$ is the outof-phase component. The out-of-phase component is of great importance since it plays the role of the aerodynamic damping, while the in-phase component plays the role of the aerodynamic stiffness. When the out-of-phase component is positive, it acts as negative aerodynamic damping indicating that self-excited oscillation may take place.

\section{Computation of the flutter derivatives}

Flutter derivatives are non-analytical parameters which relate motion-induced forces and the velocities and displacements of the structure. As a consequence, these parameters have been traditionally identified using wind tunnel tests, and more recently from numerical based simulations.

According to Sarwar and Ishihara [15], the aeroelastic forces on a bridge deck, considering two degrees of freedom (heave and pitch) can be written as follows, using Scanlan's formulation: 


$$
\begin{gathered}
L_{a e}^{S}(t)=\frac{1}{2} \rho U^{2} B\left[K H_{1}^{*} \frac{\dot{h}(t)}{U}+K H_{2}^{*} \frac{B \dot{\alpha}(t)}{U}+K^{2} H_{3}^{*} \alpha(t)\right. \\
\left.+K^{2} H_{4}^{*} \frac{h(t)}{B}\right] \\
M_{a e}^{S}(t)=\frac{1}{2} \rho U^{2} B^{2}\left[K A_{1}^{*} \frac{\dot{h}(t)}{U}+K A_{2}^{*} \frac{B \dot{\alpha}(t)}{U}+K^{2} A_{3}^{*} \alpha(t)\right. \\
\left.+K^{2} A_{4}^{*} \frac{h(t)}{B}\right]
\end{gathered}
$$

where $L_{a e}^{S}(t)$ is the aeroelastic lift force per unit of span length, $M_{a e}^{S}(t)$ is the aeroelastic moment per unit of span length, $K=(B \omega) / U$ is the reduced frequency, $h(t)$ is the heave oscillation and $\dot{h}(t)$ is its time derivative, $\alpha(t)$ in the torsional rotation and $\dot{\alpha}(t)$ its time derivative, $H_{i}^{*}$ and $A_{i}^{*}(i=1, \ldots, 4)$ are the flutter derivatives.

Assuming prescribed harmonic forced oscillations $h=h_{0} \sin \left(\omega_{h} t\right)$ and $\alpha=\alpha_{0} \sin \left(\omega_{\alpha} t\right)$, where $h_{0}$ and $\alpha_{0}$ are the amplitudes of the oscillations, and also that motion-induced forces are linear functions of the movement; after some manipulation, the following expressions are obtained for the identification of the flutter derivatives:

$$
\begin{array}{lll}
H_{1}^{*}=\frac{-L_{0} \sin \phi_{L}}{q K^{2} h_{0}} & \text { (11.a) } & A_{1}^{*}=\frac{-M_{0} \sin \phi_{M}}{q B K^{2} h_{0}} \\
H_{2}^{*}=\frac{-L_{0} \sin \phi_{L}}{q B K^{2} \alpha_{0}} & \text { (11.b) } & A_{2}^{*}=\frac{-M_{0} \sin \phi_{M}}{q B^{2} K^{2} \alpha_{0}} \\
H_{3}^{*}=\frac{L_{0} \cos \phi_{L}}{q B K^{2} \alpha_{0}} & (11 . \mathrm{c}) & A_{3}^{*}=\frac{M_{0} \cos \phi_{M}}{q B^{2} K^{2} \alpha_{0}} \\
H_{4}^{*}=\frac{L_{0} \cos \phi_{L}}{q K^{2} h_{0}} & \text { (11.d) } & A_{4}^{*}=\frac{M_{0} \cos \phi_{M}}{q B K^{2} h_{0}}
\end{array}
$$

where $L_{0}$ and $M_{0}$ are the amplitudes of the fluctuating lift and moment acting of the bluff-body, $\phi_{L}$ and $\phi_{M}$ are the phase lags of the aeroelastic lift or moment with respect to the prescribed oscillation and $q$ is the dynamic pressure.

\section{Geometry, numerical modelling and model validation}

A rectangular section with a width to depth ratio $B / H=4$ and sharp edges has been chosen as the case study for computing its forced oscillation response.

The flow domain size adopted in all the simulation reported herein is 41B by $30.25 \mathrm{~B}$. As boundary conditions, a constant velocity inlet has been set at the left side while a pressure outlet at atmospheric pressure has been imposed at the right side. The upper and lower boundaries have been defined as slip walls. A turbulence intensity of $1 \%$ has been chosen along with a $0.1 \mathrm{~B}$ turbulent length 
scale for the incoming flow. The simulations have been conducted using the open source software OpenFoam. The URANS and turbulence model equations are discretized with second order central finite difference schemes except for the convention terms for which a linear upwind scheme was selected. The Euler first order bounded implicit scheme was set for the first time derivative terms.

A 2D block structured regular mesh has been generated taking special care in the definition of the refined grid around the deck cross-section in order to obtain target values for the non-dimensional first grid height $\left(y^{+}=\left(\delta_{1} u_{*}\right) / v\right.$, where $\delta_{1}$ is the height of the first prismatic grid layer around the deck and $u_{*}$ is the friction velocity) close to 1 . In this manner, no wall functions are required.

A grid independence study has been conducted for three different grids namely Coarse, Medium and Fine grids, for the static rectangular cylinder with $0^{\circ}$ angle of incidence The Reynolds number of the simulations reported herein is about $10^{5}$. In table 1 , the data of these three grids, along with the Strouhal number and the mean values and standard deviations of the force coefficients are reported. All the computations have been carried out imposing a maximum Courant number of 1 .

The definition of the force coefficients in this work is the following:

$$
C_{D}=\frac{D}{\frac{1}{2} \rho U^{2} B} \quad C_{L}=\frac{L}{\frac{1}{2} \rho U^{2} B} \quad C_{M}=\frac{M}{\frac{1}{2} \rho U^{2} B^{2}}
$$

In the former expressions, $D$ is the drag force per span length, positive windward, $L$ is the lift force per span length, positive upwards, and $M$ is the twist moment per unit of span length, positive in the clock-wise direction. The standard deviation of the force coefficients is identified with the prime symbol in the following.

Table 1: Grid refinement study values and validation.

\begin{tabular}{|c|c|c|c|c|c|c|}
\hline Grid & Tot. cells & $S_{t}$ & $C_{D}$ & $C_{D}^{\prime}$ & $C_{L}^{\prime}$ & $C_{M}^{\prime}$ \\
\hline Coarse & 104000 & 0.145 & 0.31 & 0.009 & 0.27 & 0.046 \\
\hline Medium & 147600 & 0.145 & 0.31 & 0.012 & 0.28 & 0.046 \\
\hline Fine & 235200 & 0.145 & 0.31 & 0.011 & 0.29 & 0.045 \\
\hline Experiments [16] & & 0.159 & 0.348 & 0.081 & 0.289 & 0.054 \\
\hline
\end{tabular}

Table 1 shows very similar results for the three grids considered, which highlights the independency of the solution to the mesh density. For the simulations hereafter the Medium grid has been retained since it offers similar accuracy to the Fine mesh with lower computational cost and its extra spatial resolution with respect to the Coarse mesh offers additional guaranties for the more demanding fluid-structure interaction simulations to be addressed in the present research. It is also plain that the numerical results reported herein are similar to the experimental ones reported in [16]. 


\section{Vortex shedding response}

The forced oscillation response of the $\mathrm{B} / \mathrm{H}=4$ rectangular cylinder has been computed. The amplitude of the oscillation is $h_{0} / D=0.02$ as in the experimental tests conducted by Washizu et al. [17]. These forced oscillation simulations with constant flow speed and at different frequencies allow the identification of the reduced velocity regions where vortex-induced vibration can take place. The change in the out-of-phase component of the unsteady lift force from negative to positive values indicates that vortex-induced vibrations will occur due to the negative aerodynamic damping introduced in the system for positive values of $C_{L I}$.

In figure 1 the out-of phase component of the lift coefficient computed by the authors is presented and compared with the experimental results reported in [17] and the numerical simulations in [11] and [15]. The numerical simulation has allowed an accurate identification of the vortex induced prone region of reduced velocities for the ratio 4 rectangular cylinder.

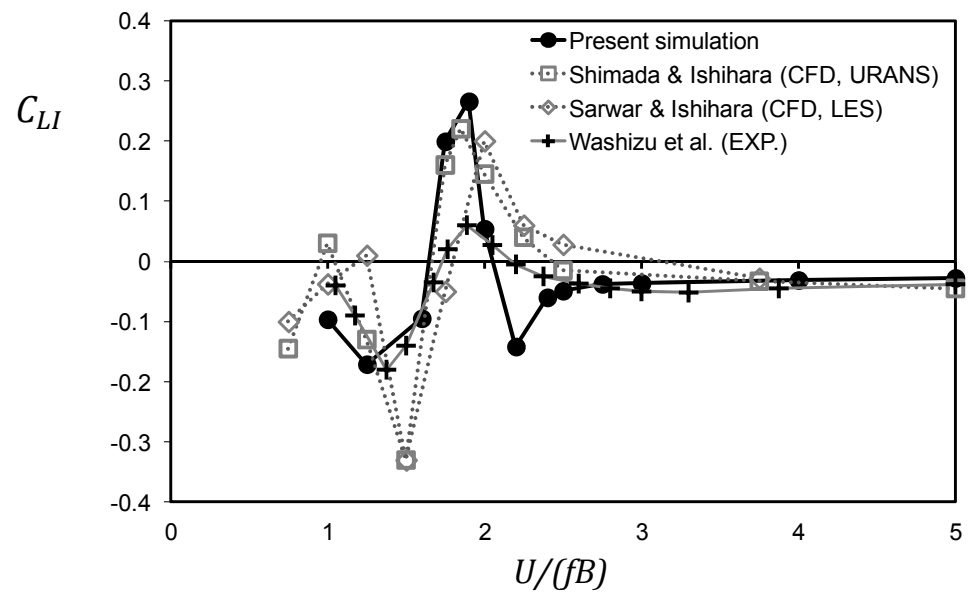

Figure 1: Out-of-phase component of the lift coefficient.

\section{Flutter derivatives computation}

The flutter derivatives of the ration 4 rectangular cylinder have been computed by means of forced oscillation simulations in heave and pitch degrees of freedom. In order to avoid the VIV prone region at a reduced velocity value close to 2, the ranges of reduced velocities considered in these simulations have been: for the heave-related flutter derivatives $H_{1}^{*}, H_{4}^{*}, A_{1}^{*}$ and $A_{4}^{*}$ the range of reduced velocities considered is $(3,6)$ as in [10], while for the pitch-related $H_{2}^{*}$, $H_{3}^{*}, A_{2}^{*}$ and $A_{3}^{*}$ flutter derivatives the range of reduced velocities is $(2,7)$.

In figure 2 four of the computed flutter derivatives are reported and compared with experimental results in [18] and numerical simulations in [10]. 

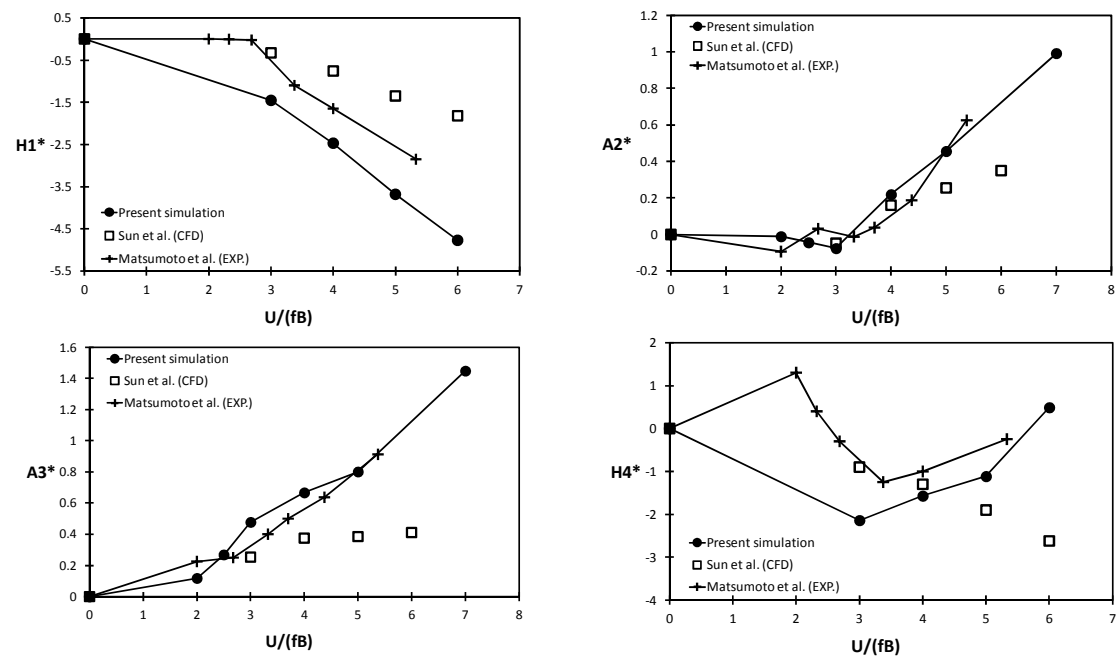

Figure 2: Flutter derivatives of the $\mathrm{B} / \mathrm{D}=4$ rectangular cylinder.

The agreement of the numerical simulations reported in this work with the experimental values available in the literature is reasonable, particularly bearing in mind the intrinsic limitation of the 2D URANS approach adopted. In fact, the numerical results approximate with more accuracy the experimental data than previous simulations by Sun and co-workers, arguably due to the bigger flow domain and the higher density mesh employed in the present simulation.

\section{Conclusions}

A 2D URANS approach using a block-structured mesh and Menter's SST k- $\omega$ turbulence model has been applied to the study of the fluid-structure interaction of a $\mathrm{B} / \mathrm{H}=4$ rectangular cylinder. The aeroelastic phenomena of interest have been VIV and the computation of flutter derivatives. A single set of heave and pitch forced oscillations has been considered for computing the responses of interest.

The reasonable accuracy of the numerical results obtained, compared with the available experimental data, has proved the feasibility of the proposed approach for identifying fluid-structure interaction responses, avoiding burdensome 3D LES simulations or the development of in-home sophisticated software. Therefore, this methodology is ready for being applied in the frame of parametric studies of bridge deck sections and, in the future, in the shape optimization of bridge decks considering aeroelastic constraints.

\section{Acknowledgements}

This work has been funded by the Spanish Ministry of Education, Culture and Sport under the Human Resources National Mobility Program of the R-D+i 
National Program 2008-2011, extended by agreement of the Cabinet Council on October $7^{\text {th }} 2011$.

The authors are grateful for access to the University of Nottingham High Performance Computing Facility and the Breogán Cluster at the University of La Coruña.

\section{References}

[1] Takeuchi, T., Matsumoto, M. (1992) Aerodynamic response characteristics of rectangular cylinders in tandem arrangement. Journal of Wind Engineering and Industrial Aerodynamics; 41-44, 565-575.

[2] Shimada, K., Ishihara, T. (2002) Application of a modified k- $\varepsilon$ model to the prediction of aerodynamic characteristics of rectangular cross-section cylinders. Journal of Fluids and Structures; 16(4), 465-485.

[3] Bruno, L., Fransos, D., Coste, N., Bosco, A. (2010) 3D flow around a rectangular cylinder: a computational study. Journal of Wind Engineering and Industrial Aerodynamics; 98, 263-276.

[4] Murakami, S., Mochida, A., Sakamoto, S. (1997) CFD analysis of windstructure interaction for oscillating square cylinders. Journal of Wind Engineering and Industrial Aerodynamics; 72, 33-46.

[5] Tamura, T. (1999) Reliability on CFD estimation for wind-structure interaction problems. Journal of Wind Engineering and Industrial Aerodynamics; 81, 117-143.

[6] Tamura, T., Itoh, Y. (1999) Unstable oscillation of rectangular cylinder at various mass ratios. Journal of Aerospace Engineering; 12(4), 136-144.

[7] Tamura, T., Ono, Y. (2003) LES analysis on aeroelastic instability of prisms in turbulent flow. Journal of Wind Engineering and Industrial Aerodynamics; 91, 1827-1846.

[8] Mendes, P.A., Branco, F.A. (1999) Analysis of fluid-structure interaction by an arbitrary Lagrangian-Eulerian finite element formulation. International Journal for Numerical Methods in Fluids; 30, 897-919.

[9] Sun, D., Owen, J.S., Wright, N.G., Liaw, K.F. (2008) Fluid-structure interaction of prismatic line-like structures, using LES and block-iterative coupling. Journal of Wind Engineering and Industrial Aerodynamics; 96, 840-858.

[10] Sun, D., Owen, J.S., Wright, N.G. (2009) Application of the k- $\omega$ turbulence model for a wind-induced vibration study of 2D bluff bodies. Journal of Wind Engineering and Industrial Aerodynamics; 97, 77-87.

[11] Shimada, K., Ishihara, T. (2012) Predictability of unsteady twodimensional $\mathrm{k}-\varepsilon$ model on the aerodynamic instabilities of some rectangular prisms. Journal of Fluids and Structures; 28, 20-39.

[12] Wilcox, D.C. (2006) Turbulence modeling for CFD. 3rd Edition; DCW Industries, Inc.

[13] Menter, F., Esch, T. (2001) Elements of industrial heat transfer prediction. 16th Brazilian Congress of Mechanical Engineering. 
[14] Sarkic, A., Fisch, R., Hoffer, R. Bletzinger, K. (2012) Bridge flutter derivatives based on computed, validated pressure fields. Journal of Wind Engineering and Industrial Aerodynamics; 104-106, 141-151.

[15] Sarwar, M.W., Ishihara, T. (2010) Numerical study on suppression of vortex-induced vibrations of box girder bridge section by aerodynamic countermeasures. Journal of Wind Engineering and Industrial Aerodynamics; 98, 701-711.

[16] Vairo, G. (2003) A numerical model for wind loads simulation on longspan bridges. Simulation Modelling Practice and Theory; 11,315-351.

[17] Washizu, K., Ohya, A., Otsuki, Y., Fujii, K. (1978) Aeroelastic instability of rectangular cylinders in heaving mode. Journal of Sound and Vibration; 59(2), 195-210.

[18] Matsumoto, M., Yagi, T., Tamaki, H., Tsubota, T. (2008) Vortex-induced vibration and its effect on torsional flutter instability in the case of $\mathrm{B} / \mathrm{D}=4$ rectangular cylinder. Journal of Wind Engineering and Industrial Aerodynamics; 96, 971-983. 